\title{
The Effect of Photon Source on Heterogeneous Photocatalytic Oxidation of Ethanol by a Silica-Titania Composite
}

\author{
Janelle L. Coutts ${ }^{\mathrm{a}}$, Lanfang H. Levine ${ }^{\mathrm{a} *}$, Jeffrey T. Richards ${ }^{\mathrm{a}}$, David W. Mazyck ${ }^{\mathrm{b}}$
}

${ }^{a}$ Team QNA - Engineering Services Contract, Sustainable Systems Applied Research, Kennedy Space Center, Florida, 32899, USA

${ }^{b}$ University of Florida, Department of Environmental Engineering Sciences, Gainesville, FL 32611, USA

* Corresponding Author at: Team QNA-ESC, Mail Code: ESC-53, Kennedy Space Center, FL 32899. Tel: 321-861-2931

Email Address: langfang.h.levine@,nasa.gov 


\title{
The Effect of Photon Source on Heterogeneous Photocatalytic Oxidation of Ethanol by a Silica-Titania Composite
}

\begin{abstract}
:
\end{abstract}
The objective of this study was to distinguish the effect of photon flux (i.e., photons per unit time reaching a surface) from that of photon energy (i.e., wavelength) of a photon source on the silica-titania composite (STC)-catalyzed degradation of ethanol in the gas phase. Experiments were conducted in a bench-scale annular reactor packed with STC pellets and irradiated with either a UV-A fluorescent black light blue lamp $\left(\lambda_{\max }=365 \mathrm{~nm}\right)$ at its maximum light intensity or a UV-C germicidal lamp $\left(\lambda_{\max }=254 \mathrm{~nm}\right)$ at three levels of light intensity. The STC-catalyzed oxidation of ethanol was found to follow zero-order kinetics with respect to $\mathrm{CO}_{2}$ production, regardless of the photon source. Increased photon flux led to increased EtOH removal, mineralization, and oxidation rate accompanied by lower intermediate concentration in the effluent. The oxidation rate was higher in the reactor irradiated by UV-C than by UV-A (38.4 vs. $31.9 \mathrm{nM} \mathrm{s}^{-1}$ ) at the same photon flux, with similar trends for mineralization (53.9 vs. 43.4\%) and reaction quantum efficiency (i.e., photonic efficiency, 63.3 vs. $50.1 \mathrm{nmol} \mathrm{CO}_{2} \mu \mathrm{mol}$ photons $^{-1}$ ). UV-C irradiation also led to decreased intermediate concentration in the effluent compared to UV-A irradiation. These results demonstrated that STC-catalyzed oxidation is enhanced by both increased photon flux and photon energy.

Keywords: Photocatalytic Oxidation, Photon Energy, Photon Source, Silica-Titania Composite (STC), Volatile Organic Compound (VOC)

1. Introduction:

Increasing awareness of health risks associated with poor air quality in closedenvironment habitats (e.g., airplanes, spacecrafts, office buildings, factories, homes, etc.) as well as increasing desire for energy conservation have provoked a high demand for 
more efficient and environmentally-friendly technologies for air revitalization. The current technology uses two major types of air purification units; the first category includes units based on filters to remove particulate matter or a sorbent material to collect gases and odors while the second category utilizes thermal oxidation whereby trace contaminants are broken down by heat with or without the assistance of a catalyst. While effective at the removal of volatile organic compounds (VOCs), these methods both have their own shortcomings. Sorbent materials and filters only trap the contaminants and must undergo further handling and disposal procedures to render the contaminants nonhazardous; they also require replacement or refurbishment after the material is spent $[1,2]$. On the other hand, thermal methods act to break down contaminants but require significant energy input for heating: temperatures in the range of $200-250^{\circ} \mathrm{C}$ for processes incorporating catalysts [3] and a range of $730-850^{\circ} \mathrm{C}$ for those processes not incorporating catalysts [4]; furthermore, there is the potential for harmful side-product formation (e.g., $\mathrm{NO}_{\mathrm{x}}$ and $\mathrm{SO}_{2}$ ) from the thermal process which requires subsequent purification [5]. An emerging alternative method for air pollution control employs the use of semiconductors in photocatalytic oxidation (PCO) of organic contaminants to produce innocuous $\mathrm{CO}_{2}$ and $\mathrm{H}_{2} \mathrm{O}[1,6,7]$. The primary advantages of $\mathrm{PCO}$ over the aforementioned technologies are the use of non-expendable materials and low energy demand because the process operates at or near room temperature.

In the photocatalytic process, light acts as an excitation source to promote an electron from the valence band to the conduction band, generating an electron-hole pair in the semiconductor catalyst. The electron and hole then participate in the reduction and oxidation of the contaminant species in a series of radical reactions [8]. The amount of 
energy required to produce the electron-hole pair is known as band-gap energy; when this energy is known, the corresponding wavelength of light can be derived from the PlanckEinstein Equation, $\mathrm{E}=\mathrm{hc} / \lambda$. Among the photocatalysts used, titanium dioxide $\left(\mathrm{TiO}_{2}\right)$ is the most widely implemented because it is inexpensive, nonhazardous, and chemically inert. Commercially available nanoparticle $\mathrm{TiO}_{2}$, known as Degussa $\mathrm{P} 25$, is a simple mixture of anatase (70-85\%), rutile, and amorphous (minor) titania [9] and has demonstrated high PCO activity in numerous studies $[8,10-14]$. The anatase phase is known for its superiority in photocatalytic activity over the rutile phase [15]. The band gap energy of anatase $\mathrm{TiO}_{2}$ is $3.2 \mathrm{eV}$; thusly, a light source with a wavelength below 388 nm has sufficient energy to activate the anatase $\mathrm{TiO}_{2}$. The question arises as to how the wavelength of a photon source below this critical value affects the photocatalytic activity of $\mathrm{TiO}_{2}$.

Previously, UV light sources of various wavelengths ranging between $250-400$ $\mathrm{nm}$, and with various intensities, have been used in $\mathrm{TiO}_{2}$-catalyzed photocatalysis $[1,11$, 16-20]. Studies by Stokke et al. [11], Dijkstra et al. [16], Cen et al. [17], Alberci and Jardin [18], Kim and Hong [19], and Jacboy [20] reported that a UV-C-irradiated ( $\lambda_{\max }=$ $254 \mathrm{~nm}$ ) reactor resulted in greater photocatalytic oxidation of VOCs than a reactor irradiated with UV-A light $\left(\lambda_{\max }=365 \mathrm{~nm}\right)$, implying that a shorter wavelength light source (i.e., higher energy photons) is more efficient. However, interpretation of the results from these studies on the effect of wavelength of $\mathrm{TiO}_{2}$-assisted photocatalysis is confounded with the influence of light intensity as these studies were conducted either at different light intensities or the light intensity was not well defined. It is well known that UV light intensity received at the catalyst surface dramatically affects oxidation rates $[1$, 
$13,21]$, but a more clear understanding of its effects needs to be addressed. Furthermore, there are discrepancies in the literature regarding whether the use of UV-A or UV-C light sources results in the formation of more intermediates. Although Grela and Colussi [21] clearly demonstrated that the reaction quantum yield for the photocatalytic oxidation of 3-nitrophenol in aerated, aqueous colloids of crystalline or metastable $\mathrm{TiO}_{2}$ nanoparticles was a function of photon wavelength $(254 \leq \lambda / \mathrm{nm} \leq 366)$, no similar data was available for gas-phase photocatalysis. Distinguishing the effect of UV wavelength from that of UV light intensity has profound implications in the design of an energy-efficient and lowrisk PCO reactor for the following two reasons: 1) despite the higher lighting efficiency of current UV-C lamps over that of UV-A lamps, UV-C radiation is more damaging and can cause serious skin and eye injuries from both direct and reflected radiation, and 2) both traditional UV-A and UV-C lamps contain a trace amount of highly toxic and EPAregulated mercury; light emitting diodes (LEDs) are a promising alternate light source and lighting efficiency increases with longer wavelength LED devices $(\sim 350 \mathrm{~nm})$ [22]. Therefore, the objective of this study was to distinguish the effect of photon flux (i.e., light intensity) from that of photon energy (i.e., wavelength) by exploring the photocatalytic degradation of ethanol in the gas phase by an adsorption-enhanced $\mathrm{TiO}_{2}$ photocatalyst (silica-titania composites, STCs) [12] under the illumination of UV-C and UV-A sources. Experiments were conducted in the same reactor, and the UV-C lamp was attenuated to obtain a range of photon fluxes that brackets that of the UV-A lamp.

2. Experimental:

2.1. Photocatalyst: Silica-titania composite pellets (STCs) were supplied by Sol Gel Solutions, LLC in the form of $2 \times 6 \mathrm{~mm}$ pellets. The STC was prepared by adding Degussa $\mathrm{P} 25 \mathrm{TiO}_{2}$ to a silica sol derived from the acid hydrolysis of tetraethyl 
orthosilicate (TEOS). It had a porosity of $30-40 \AA$ and contained $4 \% \mathrm{TiO}_{2}$ (4 g Degussa $\mathrm{P} 25 \mathrm{TiO}_{2}$ in $100 \mathrm{~mL}$ of TEOS silica precursor) [12]. The properties of the Degussa P25 $\mathrm{TiO}_{2}$ were not altered during the STC synthesis process. EDX analysis was completed on a JEOL JSM-7500F Field Emission Scanning Electron Microscope using LEI detection at an 8-mm working distance and demonstrated highly incorporated titania and silica (Fig. 1).

Figure 1

2.2. Light Sources and Characterization: An 8-W UV-A (F8T5) black light blue lamp (UVA BLB) from Philips (Amsterdam, Netherlands) with dimensions of $15.6 \mathrm{~mm}$ (diameter) x $304.8 \mathrm{~mm}$ (length) and radiant output of $1.4 \mathrm{~W}$ was selected as the UV-A source. An 8-W UV-C (G8T5/OF) germicidal lamp (UV-C GL) with $2.5 \mathrm{~W}$ of UV output from Sylvania (Danvers, MA) was selected as the UV-C source. The irradiance profiles at the surface of the catalyst bed for the selected light sources were determined in a dark room (ex situ) using a spectroradiometer (model OL754C, Optronics Laboratories, Orlando, FL). The light source (either the UV-A BLB or UV-C GL) was centered inside a quartz sleeve (28 mm O.D. and $25 \mathrm{~mm}$ I.D.) and placed directly on top of the integrating sphere of the spectroradiometer (Light attenuating discs with $12.7-\mathrm{mm}$ and $6.35-\mathrm{mm}$ diameters were used to avoid saturation of the detector during scanning of the UV-A and UV-C sources, respectively. The desired intensity of UV-C light was achieved by using a fine stainless-steel mesh (U.S. mesh size 16, referred to hereafter as attenuation mesh) between the quartz sleeve and lamp as a neutral density filter.

2.3. Photocatalytic Oxidation (PCO) Reactor: A custom-made annular reactor (Southern Scientific, Inc., Micanopy, FL) was used in this study and accommodated both light 
sources interchangeably. As shown in Fig. 2, the reactor was comprised of an outer Pyrex housing (38.8 mm I.D., $42.0 \mathrm{~mm}$ O.D.) and an inner quartz sleeve (25.0 mm I.D., $28.8 \mathrm{~mm}$ O.D.) with Teflon caps to create an air-tight environment; the reactor length was $15.24 \mathrm{~cm}$. Glass beads (3-mm dia.) were added to allow the STCs to be packed in the center of the reactor as well as to facilitate air distribution. The STC pellets $(14.6 \mathrm{~g})$ were then packed in the annulus space $(5.0 \mathrm{~mm})$ resulting in a bed height of $\sim 65 \mathrm{~mm}$. Temperature was controlled to $25 \pm 0.1^{\circ} \mathrm{C}$ throughout all experiments via a water jacket and a thermostated water bath. The light source (either UV-A BLB or UV-C GL) was centered in the quartz sleeve of the reactor and the entire reactor was covered in aluminum foil to avoid penetration of room light into the reactor system and to avoid accidental UV exposure of lab personnel.

Figure 2

2.4. PCO Experiments and Process Monitoring: Tests were performed in the annular reactor packed with $14.6 \mathrm{~g}$ of STC pellets under continuous illumination by either the UV-A source at its maximum light intensity or the UV-C source at three varied intensity levels. All tests were carried out in a flow-through mode with an uninterrupted $2 \mathrm{~L} \mathrm{~min}^{-1} \mathrm{CO}_{2-}$ free air $(74.7 \pm 0.8 \% \mathrm{RH})$ containing $50 \mathrm{ppm}_{\mathrm{v}}$ ethanol at $25^{\circ} \mathrm{C}$ as the test volatile organic compound (VOC) as described previously [13]. Each test was repeated a minimum of two times. The STC pellets were regenerated in-line between each test by passing a VOC-free $(74.7 \pm 0.8 \% \mathrm{RH})$ sweeping gas at $25^{\circ} \mathrm{C}$ through the reactor accompanied by UV irradiation. Both influent and effluent streams were sampled alternately every 8.45 minutes and analyzed for ethanol and its oxidation intermediates by GC/FID equipped 
with an HP Plot Q column (30 m X $0.32 \mathrm{~mm}, 20 \mu \mathrm{m}$ d.f.). The effluent stream was also directed to a $\mathrm{CO}_{2}$ analyzer for the determination of the rate of $\mathrm{CO}_{2}$ production.

\subsection{PCO Efficiency, Kinetics, and Reaction Quantum Yield: PCO performance was} quantified by EtOH removal, the measure of the removal of the test VOC regardless of it being adsorbed or oxidized at pseudo-steady state conditions, and mineralization efficiency $\left(\mathrm{X}_{\mathrm{A}}\right)$, the measure of complete oxidation of $\mathrm{EtOH}$ to $\mathrm{CO}_{2}$. These values were calculated using equations 1 and 2 , respectively, where $\mathrm{C}_{0}$ and $\mathrm{C}_{\mathrm{EtOH}}$ are the influent and effluent ethanol concentrations; $\Delta \mathrm{C}_{\mathrm{CO} 2}$ is the $\mathrm{CO}_{2}$ generated by the $\mathrm{PCO}$. The rate of the PCO of ethanol was determined based on the formation of $\mathrm{CO}_{2}$ rather than the disappearance of ethanol to prevent overestimation due to the $\mathrm{EtOH}$ adsorption to the silica-rich photocatalyst. The reaction quantum yield ( $\xi)$, or photonic efficiency, was calculated as the ratio of the photocatalytic oxidation rate to the incident photon flux as shown in equation 3.

$$
\begin{array}{ll}
\text { EtOH Removal }=\left(\mathrm{C}_{0}-\mathrm{C}_{\mathrm{EtOH}}\right) / \mathrm{C}_{0} & \text { Eqn. 1 } \\
\mathrm{X}_{\mathrm{A}}=\Delta \mathrm{C}_{\mathrm{CO} 2} /\left(2 * \mathrm{C}_{0}\right) & \text { Eqn. } 2 \\
\xi=\text { Rate of Reaction }\left(\mathrm{nM} \mathrm{s}^{-1}\right) / \text { Rate of incident photons }\left(\mu \mathrm{mol} \mathrm{s}{ }^{-1}\right) & \text { Eqn. 3 }
\end{array}
$$

3. Results and Discussion:

\subsection{Spectral Quality of the UV-A BLB and UV-C GL:}

The Philips brand UV-A BLB was selected as the UV-A source because it was found to possess the highest light intensity over alternate UV-A lamps previously tested [13]. The irradiance spectrum of the UV-A lamp (Fig. 3A) had a broad primary peak (354-388 nm) centered at $365 \mathrm{~nm}$ and an additional peak at $405 \mathrm{~nm}$ that is beyond the action spectrum of anatase $\mathrm{TiO}_{2}(\lambda<388 \mathrm{~nm})$. The UV-C GL irradiance spectrum 
contained a high-intensity, narrow peak (250-255 nm) centered at $253 \mathrm{~nm}$ along with several low-intensity peaks at 313, 365, and $405 \mathrm{~nm}$ (Fig. 3B).

\section{Figure 3}

The irradiance of the primary peak for each lamp was determined through the integration of the radiation scan with defined integration limits of $1 \%$ irradiance with respect to the value at the $\lambda_{\max }$. The minor peaks for both sources were also integrated in a similar fashion to determine their contribution to the total irradiance of the lamps. It was found that the $405 \mathrm{~nm}$ peak accounted for $0.71 \%$ of the total irradiance of the UV-A lamp; the irradiances for the 313,365 , and $405 \mathrm{~nm}$ peaks in the UV-C source were found to account for $0.42 \%, 0.89 \%$, and $1.06 \%$ of the total irradiance, respectively. Based on these results, it is not expected that these peaks had significant contribution to the energy used in the activation of the $\mathrm{TiO}_{2}$-assisted photocatalysis or possible photolysis of ethanol throughout this study. The irradiance at the surface of the catalyst, as well as the photon flux for both the UV-A source and UV-C source (with and without the neutral density filter), are shown in Table 1. The 8-W UV-C lamp had an irradiance 2.0 times higher than the 8-W UV-A source and was attenuated to obtain a range of intensities by the use of one or two layers of attenuation mesh.

\section{Table 1}

\subsection{Effect of Photon Flux of UV-C Light on STC-Catalyzed Oxidation of Ethanol:}

\section{Figure 4}

Figure 4 shows the change in carbon-normalized effluent composition over time after the introduction of contaminant flow and UV-C illumination. There were three components detected in the effluent stream: ethanol; $\mathrm{CO}_{2}$, the complete mineralization product; and 
acetaldehyde (ACD), the only quantifiable intermediate detected by the GC-FID. A carbon balance for the system further confirmed this observation. For the UV-C source under all three intensities tested, the total carbon in the effluent and adsorbed onto the STCs accounted for a minimum of $94 \%$ of carbon entering the system (data not shown), which is within the range of error associated with the system.

The photon flux at the catalyst surface had a profound effect on the rate of effluent concentration increase and effluent composition at any given time point (Fig. 4). A true steady state was not attainable under the time restrictions of the experiments; thus, the pseudo-steady state, or time at which $\mathrm{CO}_{2}$ formation reached a steady state and the change in effluent ethanol and ACD had reached a minimum, was implemented. The pseudo-steady state was achieved approximately after the ten-hour mark in all experiments. The average concentration between 10 and 20 hours was used to calculate the ethanol removal and mineralization efficiency. The concentration of components in the effluent stream is dependent upon the balance between their production and adsorption affinity to the STC pellets. The time it took for the initial appearance of each component in the effluent as well as the time to $50 \%$ of respective concentration at pseudo-steady state are good indicators for their affinity to STC pellets. $\mathrm{CO}_{2}$ reached its $50 \%$-concentration mark in less than 45 minutes for all experiments after the initiation of the EtOH-contaminated air flow; this suggests minimal, if any, adsorption of $\mathrm{CO}_{2}$ to the STC pellets. In the cases of ACD and $\mathrm{EtOH}$, this mark was attained within 5 hours and 8.5 hours, respectively. These results show lower adsorption affinity for ACD than for EtOH. Because of the low affinity for $\mathrm{CO}_{2}$, its rate of evolution was used to determine the PCO rate. The rate of ethanol oxidation by STC-assisted photocatalysis was 
determined to be zero-order, regardless of the UV-C irradiance level implemented with respect to $\mathrm{CO}_{2}$ evolution.

Figure 5

In general, increasing the photon flux at the catalyst surface resulted in an increase in ethanol removal (Fig. 5A), ethanol mineralization (Fig. 5B), and PCO Rate (Fig. 5C). However, the reaction quantum yield decreased with the increase of photon flux (Fig. 5D). The relationship between the photon flux $(\varphi)$ and PCO rate (r) followed an exponential trend $\left(\mathrm{r}=49.117 \varphi^{0.489}\right)$ over the range of intensities studied. Previous reports proposed that the dependence of the PCO reaction rate (r) on the photon flux $(\varphi)$ follows a first-order kinetic trend $(\mathrm{r}=\mathrm{K} \varphi$, where $\mathrm{K}$ is a constant) when $\varphi$ is $0.008 \mu \mathrm{mol}$ photons $\mathrm{s}^{-1}$ or lower, but follows a half-order kinetic trend $\left(\mathrm{r}=\mathrm{K} \varphi^{0.5}\right)$ when $\varphi$ exceeds this photon flux [23]. Since the photon flux employed in this study was in the range of 0.337-0.892 $\mu \mathrm{mol}$ photons $\mathrm{s}^{-1}$, our results are in close agreement with the relationship proposed by Egerton and King [23]. There were several differences between our system and that used by Egerton and King [23] including 1) the use of the Degussa P25 $\mathrm{TiO}_{2}$ opposed to the $100 \%$ rutile $\mathrm{TiO}_{2}$ used by Egerton and King [23] where the crystal structure may have played a role in the kinetics differently; 2) the use of a UV-C light source, and thusly, higher-energy photons (only UV-A light sources were tested previously); and 3) the use of STC instead of a $\mathrm{TiO}_{2}$ thin film: the former is not only much thicker $(5 \mathrm{~mm})$ than the latter, it also contains less $\mathrm{TiO}_{2}$ for the same surface area exposed to the light (Fig. 1). Regardless of these changes, the same relationship was developed. Furthermore, our results indicate that the relationship developed between photon flux and PCO rate by Egerton and King [23] is independent of wavelength. 
As a result of the decreased dependency of the PCO rate on photon flux within the range tested, the photonic efficiency decreased as the photon flux increased (Fig. 5D). This result implies that not all of the charge carriers generated in this range of photon flux were utilized in the redox process; furthermore, less reactive carriers may have accumulated and undergone recombination. In other words, energy-use efficiency decreases at a significantly large photon flux even though it leads to increased mineralization (Fig. 5B) and reduced intermediate evolution (Fig. 5E). A balance between energy-use efficiency and PCO efficiency must be scrutinized in the design of such PCO reactors.

The PCO of ethanol on a $\mathrm{TiO}_{2}$ surface is known to follow two similar pathways that include various intermediates such as acetaldehyde, acetic acid, formaldehyde, and formic acid [24]. As previously stated, acetaldehyde was the only intermediate detected in our system. This does not mean that acetic acid, formic acid, and formaldehyde did not form during our reaction but suggests that they were oxidized at the same (or faster) rate they were formed. Therefore, a simplified schematic of the reaction, shown below, was used to better understand the decreased evolution of acetaldehyde with increased photon flux (Fig. 5E).

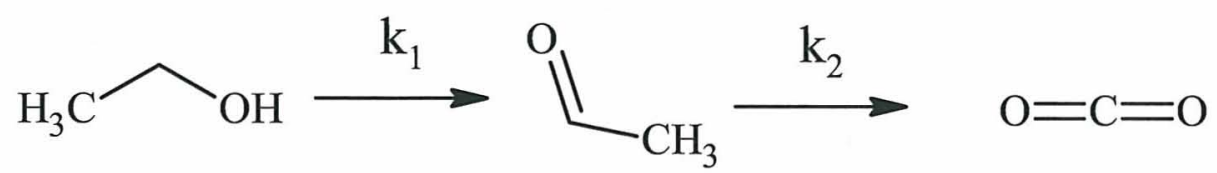

The fact that there was a significant amount of ACD in the effluent suggests that $\mathrm{k}_{2}$ is slower than $\mathrm{k}_{1}$; i.e., the oxidation of acetaldehyde is the rate-limiting step in the mineralization of ethanol. Furthermore, it may be assumed that the adsorption of ACD onto the STCs is not affected by photon flux since the time at which the $50 \%$ pseudo- 
steady state concentration mark was reached was equivalent for the various light intensities. Although both the oxidation of ethanol to acetaldehyde $\left(\mathrm{k}_{1}\right)$ and that of acetaldehyde to $\mathrm{CO}_{2}\left(\mathrm{k}_{2}\right)$, was accelerated by increased light intensity, the ACD concentration in the effluent decreased as the photon flux increases. This implies a greater increase in $\mathrm{k}_{2}$ than $\mathrm{k}_{1}$. Therefore, this result suggests that it is possible to eliminate the accumulation of ACD if sufficient light intensity and optimized reactor design are provided.

3.3. Effect of Wavelength (UV-A BLB vs. UV-C GL) at the Same Photon Flux:

The PCO of ethanol by STCs irradiated by a UV-A light source was investigated under the same conditions in the same reactor as that used in the above UV-C experiments. The key performance parameters including ethanol removal, mineralization efficiency, PCO rate, photonic efficiency, and $[\mathrm{ACD}]_{\text {Effluent }}$ are summarized in Table 2. The corresponding performance data for a reactor illuminated with UV-C light at the equivalent photon flux of the UV-A source (i.e., $0.633 \pm 0.013$ $\mu \mathrm{mol}$ photons $\mathrm{s}^{-1}$ ) was extrapolated from the relationships obtained in Figure 5 to allow for a direct comparison.

\section{Table 2}

As seen in the UV-C studies, ACD was also the only quantifiable intermediate in the UV-A studies; however, it accumulated to a higher level than seen in any of the UV$\mathrm{C}$ experiments. The total carbon balance for the UV-A-irradiated system was $94.6 \%$ again confirming the claim that was no accumulation of other intermediates. The EtOH removal for the UV-A-illuminated reactor $(89.8 \pm 1.6 \%)$ was statistically equivalent to the projected $\mathrm{EtOH}$ removal $(89.0 \%)$ for the UV-C-illuminated reactor at the equivalent 
irradiance. However, this equivalence is not due to equivalent mineralization and PCO rate (Table 2), but is likely attributed to an accelerated $\mathrm{k}_{1}$ and reduced $\mathrm{k}_{2}$, that is, an increased oxidation of ethanol to acetaldehyde and reduced oxidation of acetaldehyde to $\mathrm{CO}_{2}$, allowing for increased accumulation of $\mathrm{ACD}$ in the UV-A-irradiated reactor.

Similar to the results from the UV-C illuminated experiments, it was found that the rate of evolution of $\mathrm{CO}_{2}$ followed a zero-order rate law when the UV-A photon source was used. The PCO rate at the equivalent photon flux was $31.9 \pm 0.7$ and 39.3 $\mathrm{nM} \mathrm{CO}_{2} \mathrm{~s}^{-1}$ for the UV-A BLB and UV-C GL, respectively. This demonstrated that photons with a shorter wavelength (or higher energy) increase the PCO rate ( $\mathrm{r}_{\mathrm{UV}-\mathrm{C}}>\mathrm{r}_{\mathrm{UV}}$ A). Moreover, the reaction quantum yield for an equivalent-photon flux UV-Cilluminated reactor was 1.25 times that of the UV-A-illuminated reactor; this is consistent with the previous findings that shorter wavelength photons render greater chemical quantum yield in crystalline $\mathrm{TiO}_{2}$ sols or metastable $\mathrm{TiO}_{2}$ [21], although the magnitude of the enhancement is dependent on the catalyst used. According to Grela et al. [25], chemical quantum yield increases significantly with an increase of excess photon energy over the bandgap energy according to $\mathrm{E}^{*}=\mathrm{E}_{\lambda}-\mathrm{E}_{\mathrm{bg}}$ and reaches a plateau at $\mathrm{E}^{*}=\sim 0.9 \mathrm{eV}$. In this study, the UV-A BLB gives an $\mathrm{E}^{*}=0.2 \mathrm{eV}$ while the UV-C GL gives $\mathrm{E}^{*}=1.7 \mathrm{eV}$ over the anatase $\mathrm{TiO}_{2}$ bandgap of $3.2 \mathrm{eV}$. Our results support the theory and predict that UV-B $(290-320 \mathrm{~nm})$ give rise to the same efficiency as UV-C.

These results suggest that a shorter wavelength light source, or photons of higher energy, has an overall positive effect on the PCO of ethanol. Taking into consideration that only $\sim 10 \%$ of UV-C light compared to $\sim 90 \%$ of UV-A light is transmitted through a single layer of $\mathrm{TiO}_{2}$ thin film [17], less catalyst surface was directly exposed to the UV-C 
photons. The enhanced performance of the shorter wavelength source is more likely to be the result of 1) increased formation of potential active species in the photocatalytic oxidation reaction [26], 2) reduced electron-hole recombination [21], 3) increased interfacial electron transfer between $\mathrm{TiO}_{2}$ particles, 4) increased electron transfer from ethanol to the hot carrier of $\mathrm{TiO}_{2}$, and/or 5) increased probability for direct photooxidation of ethanol. This last hypothesis was tested by packing the reactor with 3-mm glass beads instead of STC pellets and examining whether ethanol was degraded by UV light alone.

Figure 6

No significant difference in EtOH concentration between the influent and effluent was found during this experiment with the UV-A BLB (Fig. 6A). No $\mathrm{CO}_{2}$ or ACD above the baseline level was observed in the effluent under UV-A illumination. Conversely, in the UV-C-irradiated reactor, a small quantity of ACD (average $1.36 \mathrm{ppm}_{\mathrm{v}} \mathrm{ACD}$ ) was found in the effluent, accompanied by a small decrease in $\mathrm{EtOH}$ concentration between the influent to the effluent (Fig. 6B). Clearly, differential photooxidation by UV-C and UV-A plays a small role in ethanol mineralization and is not the main contributing mechanism for the $11.7 \%$ higher mineralization efficiency, $7.4 \%$ increased PCO rate, and more 1.25 times higher photonic efficiency seen in the UV-C PCO reactor over that in the UV-A reactor.

4. Conclusions:

This study demonstrated that both photon flux and photon energy have profound impacts on not only the PCO efficiency, but also on the energy-use efficiency and must be meticulously taken into consideration in the design of an efficient PCO reactor. 
As the photon flux increased for the UV-C source, the quantum yield decreased. In accordance with previous studies, the mineralization efficiency for ethanol and PCO reaction rate increased with the incident photon flux within the range examined. This study also demonstrated that 254-nm photons (UV-C) are 1.25 times more efficient than 365-nm photons (UV-A) for driving the STC-catalyzed degradation of ethanol in the gas phase. This is in agreement with the findings by Grela et al. [21, 26] for the oxidation of salicylate (3.8 to 6.4 times depending on substrate concentration) and 3-nitrophenol. The extent of photooxidation of ethanol in the absence of the STCs by higher energy photons $(254 \mathrm{~nm})$ was slightly higher than that of lower energy photons $(365 \mathrm{~nm})$, but not sufficient to contribute to the increase in photonic efficiency, $\mathrm{PCO}$ rate, and mineralization efficiency. It is concluded that the enhanced performance by shorter wavelength photons from the UV-C light source is due to the combined result of increased active charge carriers, reduced electron-hole combination, increased interfacial electron transfer between $\mathrm{TiO}_{2}$ particles, and also increased electron transfer from ethanol to the hot carrier of $\mathrm{TiO}_{2}$.

5. Abbreviations and Nomenclature:

$\varphi-$ Photon Flux

$\xi-$ Reaction quantum yield/Photonic efficiency

ACD - Acetaldehyde

$\mathrm{C}-$ Concentration

$\mathrm{C}_{0}$ - Influent Ethanol Concentration

$\mathrm{C}_{\mathrm{EtOH}}-$ Effluent Ethanol Concentration

$\Delta \mathrm{C}_{\mathrm{CO} 2}-\mathrm{CO}_{2}$ generated from the $\mathrm{PCO}$

EtOH - Ethanol

FL - Fluorescent Lamp

GL - Germicidal Lamp

I.D. - Internal diameter

O.D. - Outer diameter

PCO - Photocatalytic Oxidation

RH - Relative Humidity 
r - PCO Rate

STC - Silica-Titania Composite

UV - Ultra-Violet

VOC - Volatile Organic Compound

$\mathrm{X}_{\mathrm{A}}-$ Mineralization Efficiency

6. Acknowledgements: This work was funded by a Kennedy Space Center Innovative

Partnership Program (IPP) grant and NASA's Exploration Life Support Program - Air

Revitalization Element. The authors would like to thank Mr. Lawrence L. Koss for his

assistance with the PCO test bed construction by making customized parts and OPTO 22 data

logging.

7. References:

[1] J. Zhao, X. Yang, Building and Environment, 38 (2003) 645-654.

[2] E. Uhde, in: T. Salthammer (Ed), Organic Indoor Air Pollutants: Occurrence, Measurement, Evaluation, Wiley-VCH. Weinheim, Germany, 1999, pp. 3-14.

[3] Thermal and Catalytic Oxidizers: Gas and Electric Fired Systems Specifications Sheets, Hitemp Technology Corporation, Flemington, NJ, 9 Dec., $2010<$ www.hitemptech.com>.

[4] A.S.K. Warahena, Y.K. Chuah, Environ. Sci. Technol. 43 (2009) 6101-6105.

[5] C.W. Babbitt, J.M. Stokke, D.W. Mazyck, A.S. Linder, J. Chem. Technol. Biotechnol. 84 (2009) 725-737.

[6] M.R. Hoffmann, S.T. Martin, W. Choi, D.W. Bahnemannt, Chem. Rev. 95 (1995) 69-96.

[7] J. Mo, Y. Zhang, Q. Xu, J. J. Lamson, R. Zho, Atmospheric Environment, 43 (2009) 22292246.

[8] O. Carp, C.L. Huisman, A. Reller, Progress in Solid State Chemistry. 32 (2004) 33-177.

[9] B. Ohtani, O.O. Prieto-Mahaney, D. Li, R. Abe, Journal of Photochemistry and Photobiology A: Chemistry. 216 (2010) 179-182.

[10] Y. Luo, D.F. Ollis, J. of Catalysis. 163 (1996) 1-11.

[11] J.M. Stokke, D.W. Mazyck, C.Y. Wu, R. Sheahan, Environmental Prog. 25(4) (2006) 312318.

[12] J.M. Stokke, D.W. Mazyck, Env. Sci. Technol. 42(10) (2008) 3808-3813.

[13] L.H. Levine, J.R. Richards, R.R. Soler, F. Maxik, J.L. Coutts, R.M. Wheeler, $40^{\text {th }}$

International Conference on Environmental Systems, Barcelona, Spain, July 2010, AIAA Paper\# 2010-6151

[14] S. Kwon, M. Fanb, A.T. Cooper, H. Yang, Critical Reviews in Environmental Science and Technology. 38(3) (2008) 197-226.

[15] A.G. Agrios, K.A. Gray, E. Weitz, Langmuir. 19 (2003) 1402-1409.

[16] M. F. J. Dijkstra, H.J. Pannerman, J. G. M. Ninkelman, J. J. Kelly, A. A. C. M. Beenackers, Chemical Engineering Science. 57 (2002) 4895-4907.

[17] J. Cen, X. Li, M. Xe, S. Zheng, M. Feng, Chemosphere (Technical Note). 62 (2006) 810816.

[18] R. M. Alberci, N. F. Jardim, Applied Catalysis B: Environmental. 14 (1997) 55-68. 
[19] S. B. Kim, S. C. Hong, Applied Catalysis B: Environmental. 35 (2002) 305-315.

[20] W.A. Jacoby, Destruction of trichloroethylene in air via semiconductor mediated gas-solid heterogeneous photocatalysis, PhD Dissertation, Department of Chemical Engineering, University of Colorado, USA, 1993.

[21] M. A. Grela, A. J. Colussi, J. Phys. Chem. B. 103 (1999) 2614-2619.

[22] A. Sandhu, Nature Photonics. 1 (2007) 38.

[23] T. A. Egerton, C. J. King, J. Oil Col. Chem. Assoc. 62 (1979) 386-391.

[24] D. S. Muggli, J. T. McCue, J. L. Falconer, Journal of Catalysis. 173 (1998) 470-483.

[25] M. Grela, M. A. Brusa, A. J. Colussi, J. Phys. Chem. B. 101 (1997) 10986-10989.

[26] Y. Paz, in: H. I. de Lasa, B. S. Rosales (Eds.), Advances in Chemical Engineering:

Photocatalytic Technologies, Elsevier, Inc., Amsterdam, 2009, pp. 289-336.

Tables:

Table $1^{\mathrm{a}}$ : Average Irradiance and Photon Flux for Selected Light Sources

\begin{tabular}{|c|c|c|}
\hline Light Source & $\begin{array}{l}\text { Irradiance } \\
\left(\mathrm{mW} \mathrm{cm} \mathrm{cm}^{-2}\right)\end{array}$ & $\begin{array}{c}\text { Photon Flux } \\
\left(\mu \text { mol photons s }{ }^{-1}\right)\end{array}$ \\
\hline UV-A BLB & $3.49 \pm 0.07$ & $0.633 \pm 0.013$ \\
\hline UV-C GL + 2X Mesh & $2.71 \pm 0.07$ & $0.337 \pm 0.009$ \\
\hline UV-C GL + 1X Mesh & $5.17 \pm 0.07$ & $0.643 \pm 0.009$ \\
\hline UV-C GL + No Mesh & $7.17 \pm 0.07$ & $0.892 \pm 0.009$ \\
\hline
\end{tabular}

${ }^{\mathrm{a}}$ : Values are the average of three scans with standard deviation. The photon flux is calculated to reflect that reaching the surface of the catalyst.

Table $2^{\mathrm{b}}$ : Effect of Photon Energy on PCO Performance

\begin{tabular}{|c|c|c|c|c|c|}
\hline $\begin{array}{l}\text { Light } \\
\text { Source }\end{array}$ & $\begin{array}{c}\text { EtOH } \\
\text { Removal } \\
(\%)\end{array}$ & $\begin{array}{c}\text { Mineralization } \\
(\%)\end{array}$ & $\begin{array}{c}\text { PCO Rate } \\
\left(\text { nM CO }_{2} \mathrm{~s}^{-1}\right)\end{array}$ & $\begin{array}{c}\xi \xi \\
\text { (nmol } \mathrm{CO}_{2} \\
\mu m o l \text { photons) }\end{array}$ & $\begin{array}{c}\text { [ACD }]_{\text {Effluent }} \\
\left(\mathbf{p p m}_{\mathrm{v}}\right)\end{array}$ \\
\hline UV-A BLB & $89.8 \pm 1.6$ & $43.4 \pm 0.3$ & $31.9 \pm 0.7$ & $50.5 \pm 0.1$ & $19.1 \pm 0.2$ \\
\hline $\mathrm{UV}-\mathrm{C} \mathrm{GL}^{\mathrm{c}}$ & 89.0 & 55.1 & 39.3 & 63.3 & 13.8 \\
\hline
\end{tabular}

Figure Captions:

Figure 1: SEM (x 5,000 magnification, LEI detection, 8-mm W.D.) image of a crushed STC pellet; EDX image analysis revealed that the white areas corresponded to titania while the darker grey areas corresponded to silica.

Figure 2: Annular Photocatalytic Reactor Packed with $14.6 \mathrm{~g}$ STC Pellets as used in experiments.

Figure 3: Average irradiance distribution for the (A) UV-A BLB at maximum irradiance and (B) UV-C GL at maximum irradiance (solid line), attenuated irradiance using one layer of mesh (- - line), and attenuated irradiance using two layers of mesh ( $\cdots$ line). The inset in (B) shows the secondary irradiance peaks for the full-irradiance UV-C GL. 
Figure 4: Time coruse of effluent composition (carbon-normalized) during STC-catalyzed oxidation of ethanol using the (A) full-irradiance UV-C GL, (B) UV-C GL with one layer of attenuation mesh, and (C) UV-C GL with two layers of attenuation mesh. Effluent species are designated as follows: ( $\$$ : $\mathrm{CO}_{2}$ carbon, $(\Delta)$ : $\mathrm{ACD}$ carbon, and (०): EtOH carbon.

Figure 5: Relationships between photon flux and (A) Ethanol Removal, (B) Mineralization Efficiency, (C) PCO Rate Constant $\left(\mathrm{nM} \mathrm{CO}_{2} \mathrm{~s}^{-1}\right)$, and (D) Photonic Efficiency, $\xi\left(\mathrm{nM} \mathrm{CO}_{2} \mu \mathrm{mol}\right.$ photons $\left.{ }^{-1}\right)$. Data points designated with $(\circ)$ were obtained using the UV-A light source and those designated with ( $\$$ were obtained using the UV-C light source.

Figure 6: Influent $(\diamond)$ and effluent $(\diamond) \mathrm{EtOH}$ composition during photolysis of ethanol by the (A) UV-A BLB and (B) UV-C light sources.

Figures:

Figure 1:

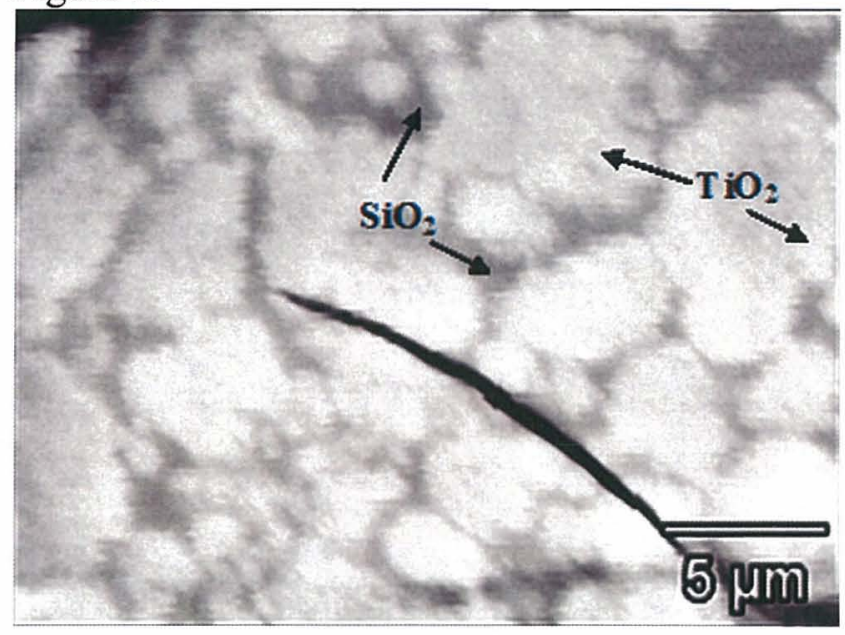

Figure 2:

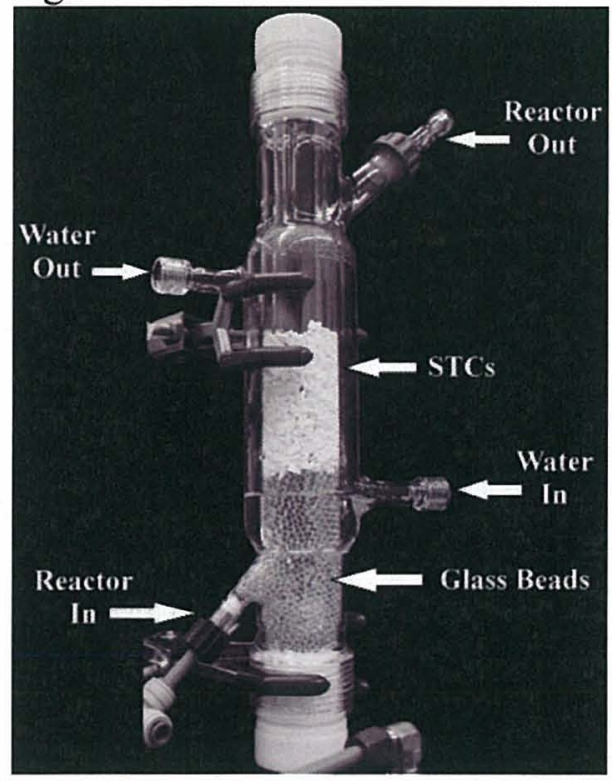


Figure 3:
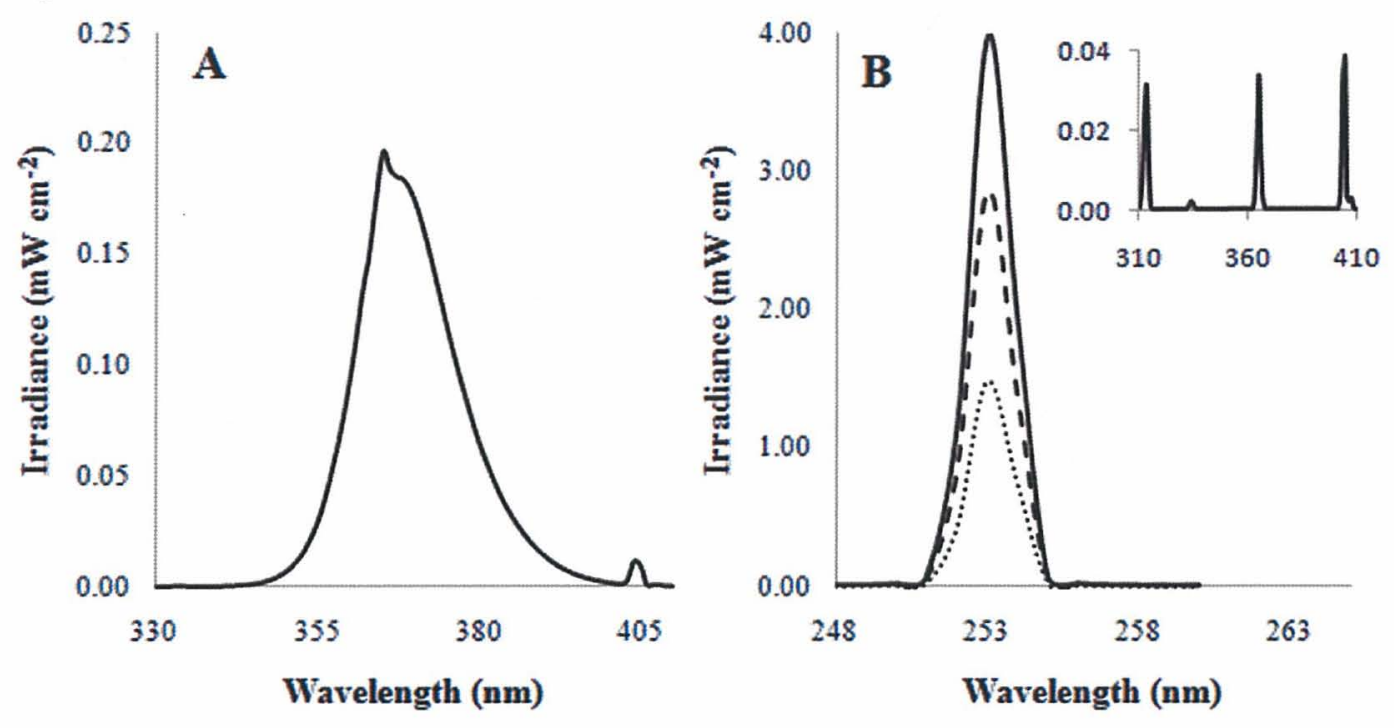

Figure 4:

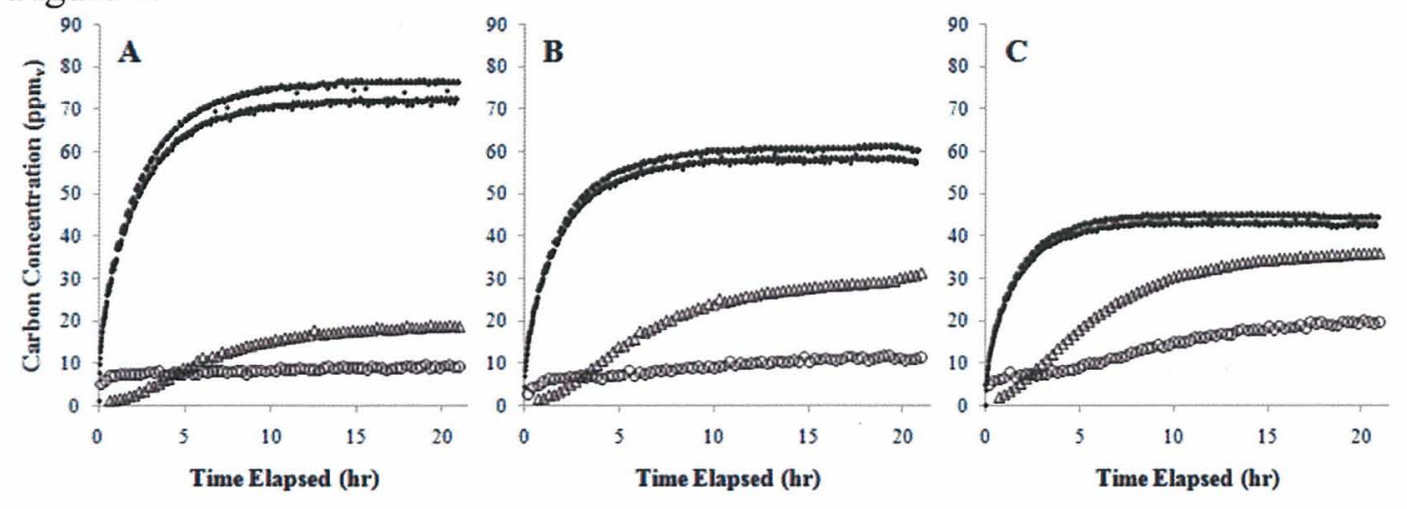

Figure 5: 

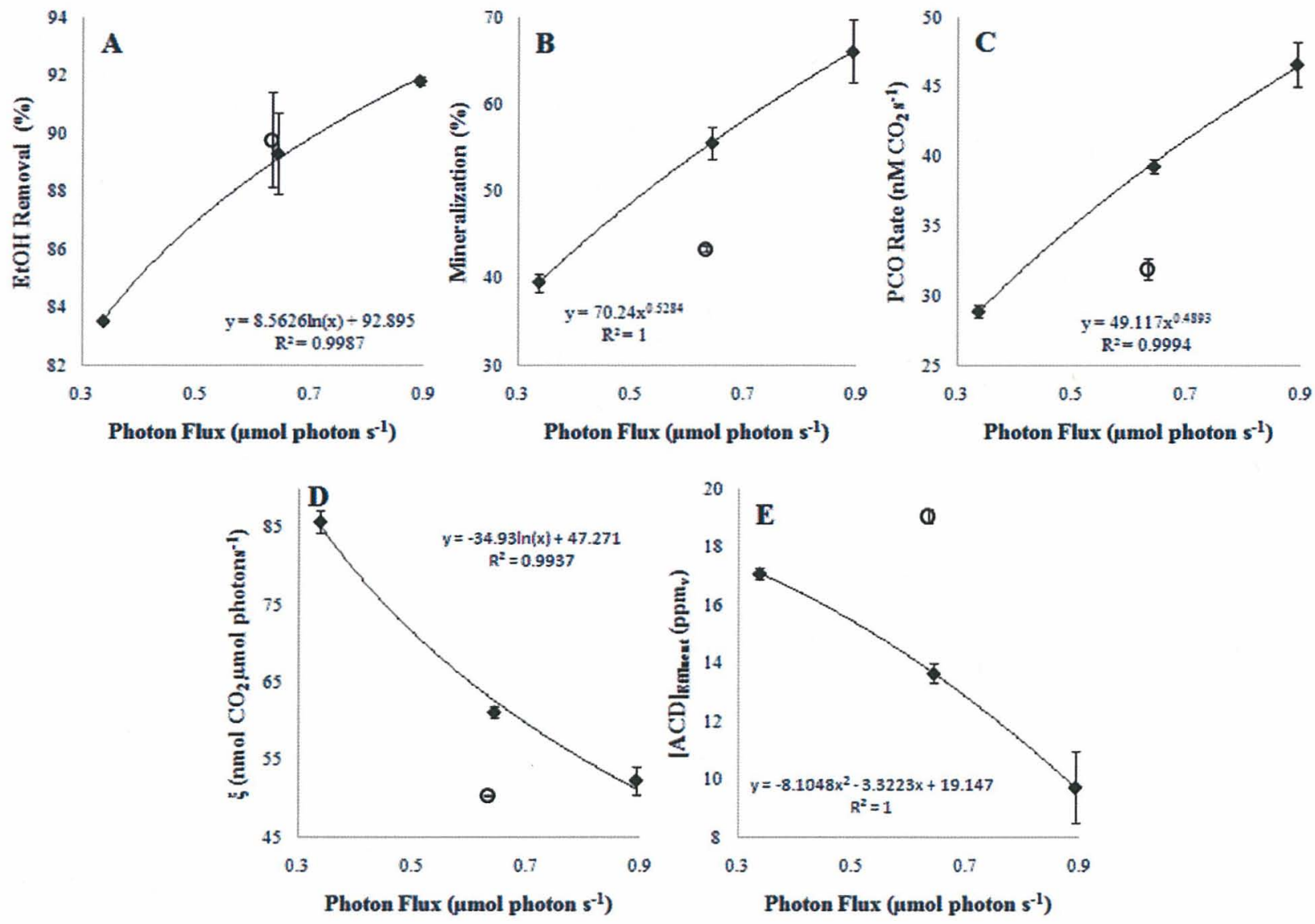

Figure 6: 

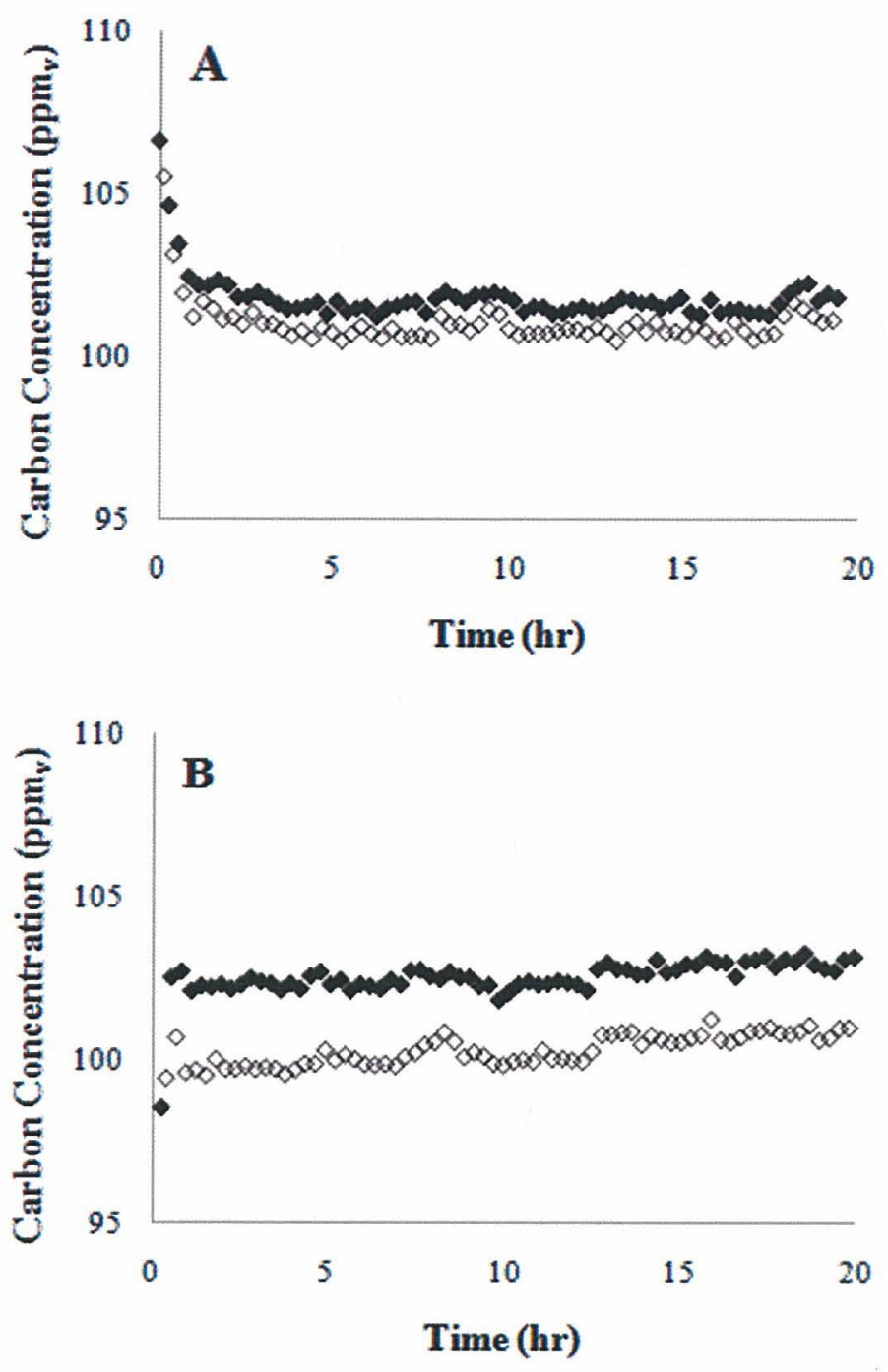